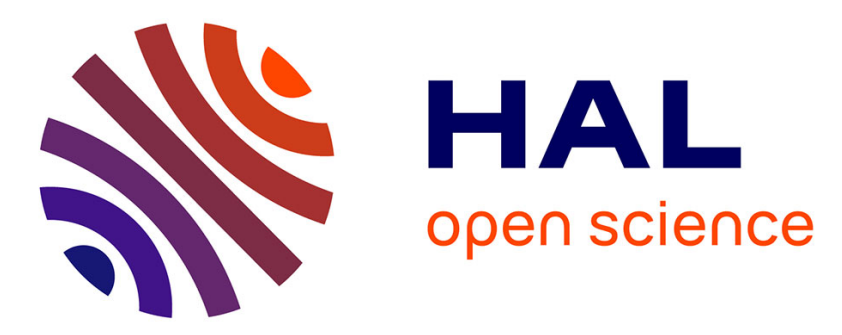

\title{
Towards accurate and reproducible predictions for prognostic: an approach combining a RRBF Network and an AutoRegressive Model.
}

Ryad Zemouri, Rafael Gouriveau

\section{- To cite this version:}

Ryad Zemouri, Rafael Gouriveau. Towards accurate and reproducible predictions for prognostic: an approach combining a RRBF Network and an AutoRegressive Model.. 1st IFAC Workshop on Advanced Maintenance Engineering, Services and Technology, IFAC A-MEST'10., Jul 2010, Lisbonne, Portugal. pp.163-168. hal-00503906

\section{HAL Id: hal-00503906 https://hal.science/hal-00503906}

Submitted on 19 Jul 2010

HAL is a multi-disciplinary open access archive for the deposit and dissemination of scientific research documents, whether they are published or not. The documents may come from teaching and research institutions in France or abroad, or from public or private research centers.
L'archive ouverte pluridisciplinaire HAL, est destinée au dépôt et à la diffusion de documents scientifiques de niveau recherche, publiés ou non, émanant des établissements d'enseignement et de recherche français ou étrangers, des laboratoires publics ou privés. 


\title{
Towards Accurate and Reproducible Predictions for Prognostic: an Approach Combining a RRBF Network and an AutoRegressive Model
}

\author{
Ryad Zemouri*, Rafael Gouriveau** \\ * Laboratoire d'Automatique du CNAM, \\ 75003 Paris, France, (e-mail: ryad.zemouri@cnam.fr) \\ ** FEMTO-ST Institute, UMR CNRS 6174 - UFC / ENSMM / UTBM, \\ Automatic Control and Micro-Mechatronic Systems Department \\ 25000 Besançon, France (e-mail: rgourive@ens2m.fr)
}

\begin{abstract}
In prognostic's field, the lack of knowledge on the behavior of equipments can impede the development of classical dependability analysis, or the building of effective physic-based models. Following that, artificial neural networks (ANNs) appear to be well suited since they can learn from data gathered from equipments. In this paper, an approach combining a Recurrent Radial Basis Function network (RRBF) and an AutoRegressive with eXogenous inputs model (ARX) is proposed in order to perform the prediction step of prognostics: the ARX attempts to correct the error of predictions of the RRBF. Moreover, since performances of an ANN can be closely related to initial parameterization of the network, a criterion is defined to quantify the reproducibility of predictions and thereby a priori estimate the usefulness of neural network structure. The whole aims at improving the prediction step of prognostics, which is critical with respects to real applicative conditions.
\end{abstract}

Keywords: Prognostic, Neural Networks, AR Model, Time-Series Prediction, Accuracy, Reproducibility.

\section{INTRODUCTION}

Although there are some divergences in literature, prognostic can be defined as proposed by the International Organization for Standardization: it is "the estimation of time to failure and risk for one or more existing and future failure modes" (ISO, 2004). It is thereby a process whose objective is to predict the remaining useful life (RUL) before a failure occurs given the current machine condition and past operation profile (Jardine et al., 2006). A central problem can be pointed out from this: the accuracy of a prognostic system is related to its ability to approximate and predict the degradation of an equipment, and the prediction phase is thereby a critical one. A wide variety of approaches can be used for that purpose (Byington et al., 2002, Vachtsevanos et al., 2006). Their applicability is however limited by classical constraints of the system under study: available data, knowledge or experiences, dynamic and complexity of the system, implementation requirements (precision, computation time, etc.), available monitoring devices. Following that, data-driven have been increasingly applied to prognostics. These approaches use real data to approximate and track features revealing the degradation of components and to forecast the global behavior of a system. Indeed, in many applications, measured input/output data is the major source for a deeper understanding of the system degradation. Within data-driven approaches, artificial neural networks (ANNs) appear to be very promising prognostic tools: they learn from examples and attempt to capture the subtle relationship among data even if the underlying relationships are unknown or hard to describe (by a learning process). Actual developments confirm the interest of using
ANNs in forecasting applications (Andalib et al., 2009, Graves et al., 2009, Pisoni et al., 2009).

In this context, the purpose of the work is to propose a nonlinear forecasting model based on ANN and to improve its prediction performances. The recurrent radial basis function network (RRBF) proposed by Zemouri et al. (2003) is presented as a candidate to ensure a first level of prediction, and an AutoRegressive with eXogenous inputs model (ARX) is used to improve its accuracy by correcting the error of predictions. Moreover, since performances of an ANN are partially depending on an initial randomly parameterization of its structure, criteria are proposed to quantify the precision, the accuracy, and the reproducibility of predictions. It enables to a priori estimate the usefulness of a neural network structure. Note that the proposition of this paper does not cover all aspects of prognostics, but only emphasizes on the prediction step. Indeed, in order to perform prognostic, one should also integrate the ways of considering various failure modes, maintenance actions, performance threshold models...

The paper is organized as follows. In section 2, the main theoretical backgrounds of RRBF networks and ARX models are presented. On this basis, the whole prediction architecture is proposed. Let call it a NARX model (Nonlinear ARX). The next part is dedicated to the proposition of measures to quantify the "quality" of NARX-based prediction is presented. The whole is finally illustrated and discussed on a mainly used prediction benchmark (the Mackey Glass time series), and on a real-world prognostics problem concerning the prediction of an engine health. 


\section{PREDICTION ARCHITECTURE}

\subsection{The Radial Basis Function Network (RBF)}

The Radial Basis Function network (RBF) is commonly used to model uncertain and nonlinear functions. That can be seen as an approximation problem in a high-dimensional space.

A RBF is a two-layers processing structure (Fig. 1.a). The hidden layer consists of an array of computing units $\left(\phi_{1}, \phi_{2}, \ldots \phi_{k}\right)$. These hidden units provide a set of basis functions of the input vectors as they are expanded into the higher dimension hidden-unit space. The mapping from the input vectors to the outputs of the hidden units is nonlinear, whereas that one from the hidden units to the final one is linear. In prediction application, the general mapping function of the RBF network can be expresses as:

$$
y(t+n)=\mathbf{g}[\mathbf{y}, \boldsymbol{w}]=\sum_{j=1}^{k} w_{p j} \phi_{j}(\mathbf{y})
$$

where $\mathbf{y}=[y(t), y(t-1), \ldots, y(t-d)]^{T}$. The second layer of a RBF network can be seen as a summation layer with a set of weights $\left(w_{p 1}, w_{p 2}, \quad, w_{p k}\right)$. The commonly used RBF basis function is the Gaussian one. With notations $\left(\mathbf{c}_{j}, \boldsymbol{\sigma}_{j}\right)$ and $\boldsymbol{x}$, the centre and width of the radial basis function, and the input vector:

$\phi_{j}(\boldsymbol{x})=\exp \left[-\left(\mathbf{x}-\mathbf{c}_{j}\right)^{2} /\left(2 \boldsymbol{\sigma}_{j}^{2}\right)\right]$

Training a RBF network with linear outputs is very fast and is accomplished through two stages.

- The first stage is unsupervised and accomplished by obtaining cluster centers of the training set input vectors. A popular method for that purpose is the kmeans clustering.

- The second stage consists in solving a set of linear equations, the solution of which can be obtained by a matrix inversion technique such as singular value decomposition or least squares method.

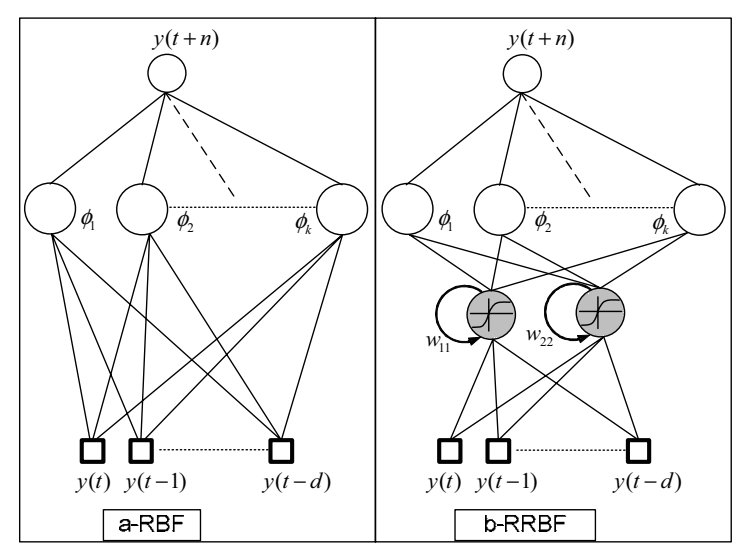

Fig. 1. a) Radial Basis Function and b) Recurrent Radial Basis Function networks, for n-step ahead predictions.

\subsection{The Recurrent Radial Basis Function Network (RRBF)}

The Recurrent RBF neural network considers time as an internal representation (Fig. 1.b). The dynamic aspect is obtained by the use of an additional self-connection to the input neurons with a sigmoid activation function. The RRBF network can thus take into account a certain past of the input signal. The output of each neuron of the input layer is the summation of the current input $x_{i}$ and its previous output weighted by a self-connection $w_{i i}$ :

$a_{i}(t)=w_{i i} \xi_{i}(t-1)+x_{i}(t), \quad \xi_{i}(t)=f\left(a_{i}(t)\right)$

where $a_{i}(t), \xi_{i}(t)$ and $f$ are the neuron activation and its output at time $t$, and the sigmoid activation function:

$f(x)=(1-\exp (-\kappa x)) /(1+\exp (-\kappa x))$

The RRBF network was described on (Zemouri et al., 2003). A mathematical study of the dynamic behavior of looped neuron can be found in (Frasconi et al., 1992). To have the longest memory, the self connection weight $w_{i i}$ and the parameter $\kappa$ must respect the relation $\kappa w_{i i}=2$.

\subsection{Combining RRBF and ARX: a NARX architecture}

RRBF appears to be a good candidate for time series prediction applications (Zemouri et al., 2003). In a general way, the prediction $\hat{y}(t+n)$ obtained by the neural network can be expressed by a residual prediction error $\varepsilon$ as:

$\varepsilon(t+n)=y(t+n)-\hat{y}(t+n)$

Considering the prediction $\hat{y}(t+n)$ obtained according to the input $y(t)$, the residual error $\varepsilon(t+n)$ can not be calculated until the time instant $(t+n)$. The prediction for $(t+n)$ can be expressed by the following expression:

$\hat{\Psi}(t+n)=\hat{y}(t+n)+f(\varepsilon(t))$

where $\varepsilon(t)$ is the residual error at the instant $t$, and $f$ is the function to find for minimizing the prediction error. In a previous work, we used a Proportional-Integral-Derivative controller for $f$ (Zemouri et al., 2009). In this paper, the prediction $\hat{y}(t+n)$ is combined with an AutoRegressive eXogenous to process the residual prediction error. The whole results in the NARX model depicted in Fig. 2. Predictions obtained through the RRBF network $(\hat{y}(t+n), \hat{y}(t), \hat{y}(t-1), \quad, \hat{y}(t-m))$ are computed with real values $(y(t), y(t-1),, y(t-m))$ and the n-step ahead prediction obtained by the whole model is:

$\hat{\Psi}(t+n)=\mathbf{F}[y, \hat{y}]=\alpha \hat{y}(t+n)+\sum_{i=0}^{i=m} a_{i} \hat{y}(t-i)+\sum_{i=0}^{i=m} b_{i} y(t-i)$

Parameters $\left(\alpha, a_{i}, b_{i}\right)$ are calculated by linear regression (matrix inversion technique).

Other types of NARX models can be found in (Andalib et al., 2009, Pisoni et al., 2009, Wei et al., 2007). 


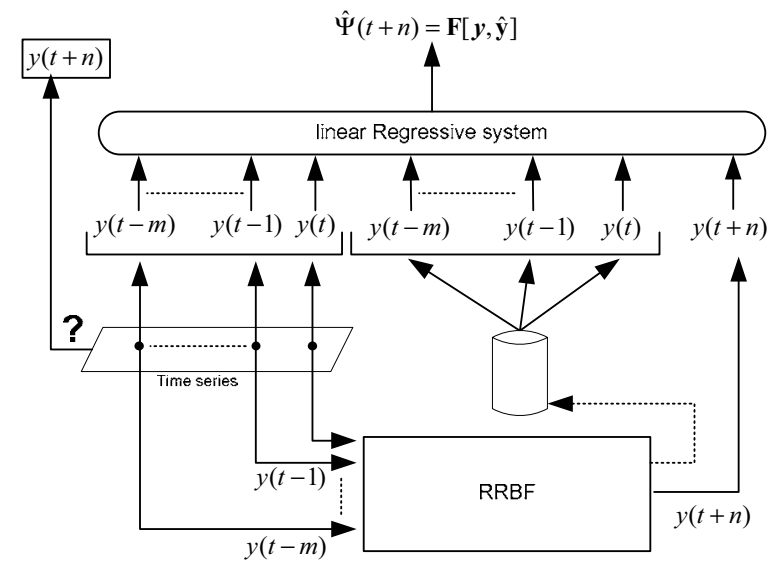

Fig. 2. The NARX architecture for n-step ahead predictions.

\section{PERFORMANCE CRITERIA FOR PREDICTION}

\subsection{Prognostics measures}

There is no general agreement as to an appropriate and acceptable set of metrics that can be employed in prognostic applications but various measures emerge from literature (Vachtsevanos et al., 2006). 1) The main objective of prognostic is to provide the efficient information that enables the choice of maintenance actions. Thus, a first set of metrics are those that quantify the risks incurred by the monitored system: the prognostic measures. The mainly pursued is the remaining useful life (RUL). 2) Assuming that prognostic is in essence an uncertain process, it is useful to be able to judge from its "quality" in order to imagine more suitable actions and prognostic system performance measures must be built. Accuracy and precision of prognostics are usually pointed out. They enable to quantify the quality of past prognostics. The second set of metrics is closely related to the prediction performances of a prognostics system. Nevertheless, this kind of metrics can be constructed when the prognostics system itself is already established. Thereby, next paragraphs aim at proposing a set of measures that can be constructed during the building phase of a prognostic system in order to a priori test the accuracy of potential prediction models.

\subsection{Prediction performance criteria}

The learning phase of a neural network can be viewed as a nonlinear optimization problem in which the goal is to find a set of network parameters minimizing a cost function for given examples: learning is an optimization process that produces an output that is as close as possible to the desired output by adjusting network parameters. That said, for a same learning set, the network structure or parameters can be different at each training run. Indeed, the learning phase starts with a random initialization of some parameters, and the quality of predictions differs at each running of the training algorithm. Thereby, the performances of a prediction model can be evaluated only if several running of the "training/test" process are processed.
Suppose that $M$ represents the number of "training/test" processes, and that $n$ designates the amount of tests in each one. For every running $i$ of the training algorithm, the mean prediction error $E(i)$ and the standard deviation $\operatorname{std}(i)$ can be expressed as follows:

$$
E(i)=\frac{1}{n} \sum_{j=1}^{n}\left(\xi^{i}(j)-\zeta(j)\right), \operatorname{std}(i)=\sqrt{\frac{1}{n} \sum_{j=1}^{n}(E(i)-\zeta(j))^{2}}
$$

where, $\xi^{i}(j)$ and $\zeta(j)$ are the $j^{\text {th }}$ output obtained by the $i^{\text {th }}$ neural model and the $j^{\text {th }}$ system output. On this basis, at least three prediction performance criteria can be built.

1) Overall Average Bias $(O A B)$ can be expressed as the mean of the $M$ values of the absolute mean prediction error $|E(i)|$ :

$$
O A B=\frac{1}{M} \sum_{i=1}^{M}|E(i)|, \quad O A B \in[0, \infty[
$$

The perfect score is $O A B=0$. This criterion enables to measure how close predictions of the $M$ potential models are to real values.

2) Overall Average Variability $(O A V)$ can be expressed as the mean of the $M$ values of the standard deviation $\operatorname{st}(i)$ :

$$
O A V=\frac{1}{M} \sum_{i=1}^{M} s t d(i), \quad O A V \in[0, \infty[
$$

The perfect score is $O A V=0$. This criterion enables to measure how close predictions of the $M$ potential models are clustered together.

3) Reproducibility (Rep) can be expressed as a mean distance in between all the $M$ prediction models performances:

$$
\operatorname{Rep}=\sqrt{\frac{2}{M(M-1)} \sum_{i<j}\left(d_{i j}\right)^{2}}, \quad \operatorname{Rep} \in[0, \infty[
$$

where $d_{i j}$ is the Euclidian distance in between the $i^{\text {th }}$ and $j^{\text {th }}$ prediction models: $\left(d_{i j}\right)^{2}=\left(E_{j}-E i\right)^{2}+(\operatorname{std}(j)-s t d(i))^{2}$. Perfect score is $R e p=0$. This criterion enables to measure how close the performances of all prediction models are grouped together, and thereby to estimate the importance of the random initialization of parameters. An illustration is proposed in Fig. 3.

\subsection{Interest criteria}

The three prediction criteria $(O A B, O A V$ and Rep) enable to judge from the performance of the prediction model and can be combined to construct a usefulness criterion. Let call it the Interest Criterion (IC) defines as follows:

$$
\left.I C=\frac{1}{O A B+O A V+R e p}, \quad I C \in\right] 0, \infty[
$$

A prediction model is as useful as its overall average bias, its overall average variability, and its reproducibility are low. Thereby, as high the interest criterion $I C$ is, as high the confidence on predictions is. Fig. 4 depicts some examples of predictions that can be obtained with their respective performance and interest criteria. 


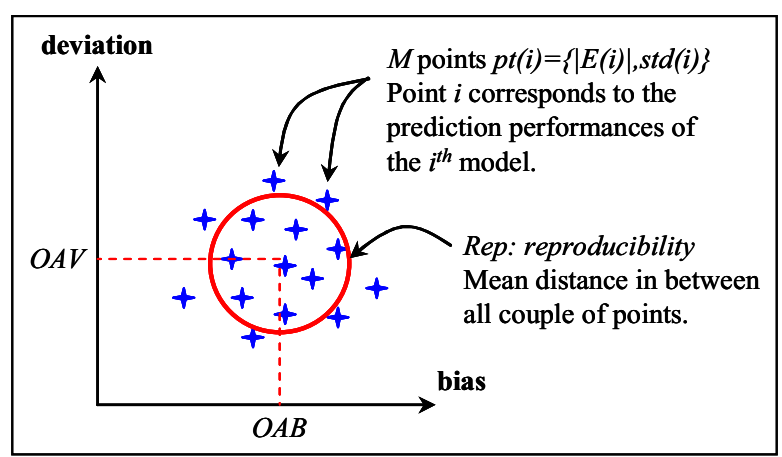

Fig. 3. Illustration of the reproducibility criterion.

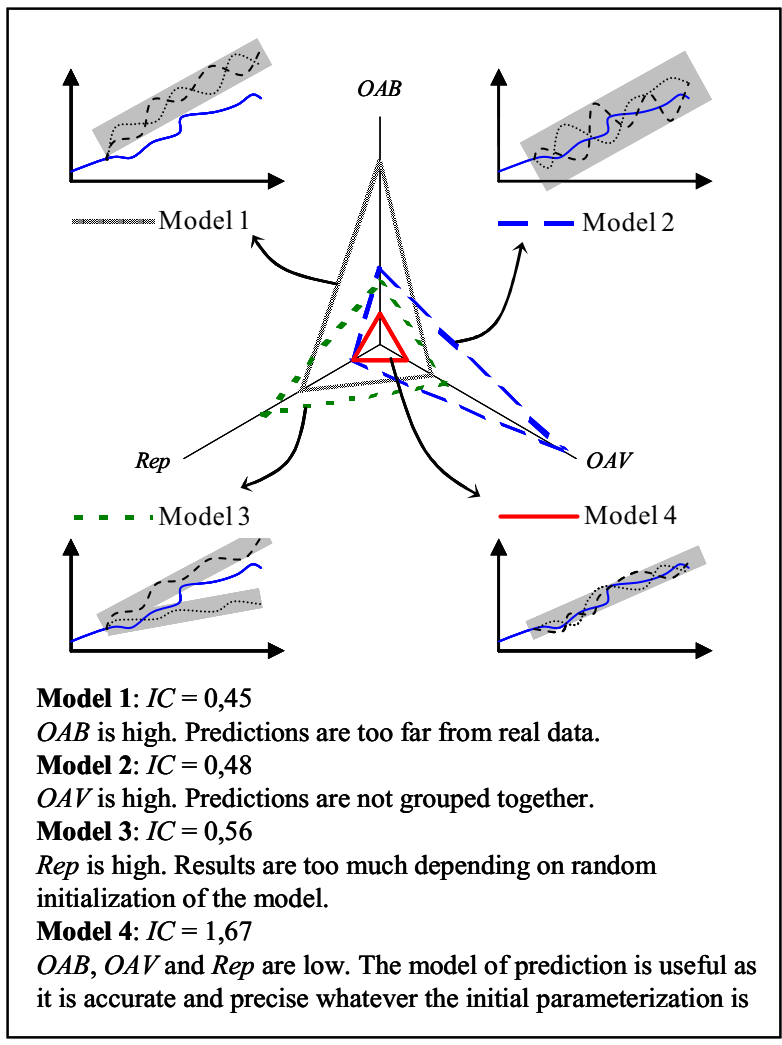

Fig. 4. Illustration of the prediction and interest criteria.

\section{EXPERIMENTS AND DISCUSSION}

\subsection{First data set: Mackey-Glass prediction benchmark}

First experiments have been made on the Mackey-Glass time series data set which is a benchmark problem extensively used: it's a non periodic and non convergent time series. Considering our final applicative objective (prognostic), to be capable to carry out predictions on such a signal is of good omen: real systems have generally a non-stationary and nonlinear behavior, what makes difficult a modeling phase. The time series is governed by eq. 13. Tests aimed at predicting future values by using past values (eq. 14).

$$
\begin{aligned}
& \frac{d y}{d t}=\beta \frac{y(t-\delta)}{1+y(t-\delta)^{\tau}}-\alpha y(t), \text { with } \alpha, \beta, \delta, \tau \in \Re \\
& y(t+n)=\mathbf{F}[y(t), y(t-1)]
\end{aligned}
$$

Three sets were used: a training, a validation, and a test set. The first set was used in order to train the parameters of the neural network (the $k$ centers of the Gaussian nodes and the output weights). In order to find the best RRBF structure with regard to the $k$ centers, several neural networks models have been created by varying the number of basis functions from 2 to 50 nodes, whereas the basis width parameter of the nodes was fixed to 1 . The $k$-means training algorithm has been used to find the best Gaussian centers for each $k$-structure. The neural network trained with the first set is validated in the second one. Validation uses data different from the training set, thus the validation set is independent from the estimated model. This helps to select the best one among the different model parameters. To avoid overfitting or underfitting, the optimal model parameters should be selected so as to have the best performance measure associated with the validation set. Since this dataset is independent from the estimated model, the generalization error obtained is a fair estimated. The model which gives the best mean prediction error in the validation set is then selected and tested in the third set (the test set). The model with the best generalization performance is then selected with the crossvalidation technique. All data have been normalized by range $[-1,+1]$. In order to measure the stability of results in time, predictions were performed from " $t+1$ " to " $t+10$ " by increments of 1 as described in (15). Once the best RRBF model is found, the second step is to find the NARX model parameters $(a, b, c, d, e)$ by linear regression on the second set (the validation set).

$$
\begin{aligned}
& \hat{\Psi}(t+n)=\mathbf{F}[\boldsymbol{y}, \hat{\mathbf{y}}] \\
& \quad=a \cdot \hat{y}(t+n)+b \cdot \hat{y}(t)+c \cdot \hat{y}(t-1)+d \cdot y(t)+e \cdot y(t-1) \\
& \hat{y}(t+n)=\mathbf{g}[(y(t), y(t-1)), \boldsymbol{w}]=\sum_{j=1}^{k} w_{j} \phi_{j}(y(t), y(t-1))
\end{aligned}
$$

Simulation test has been run 1000 times: $i=1$ to $M=1000$. At each simulation running $i$, the mean prediction error $E(i)$ and the standard deviation of the error of prediction $\operatorname{std}(i)$ were calculated in order construct the performance criteria $(9$, 10 and 11). The whole was used to evaluate the interest criterion of the model of prediction (12). Fig. 5 and 6 shows the prediction performances $(O A B, O A V$ and Rep) and the interest criterion (IC) obtained for: 1) the RRBF as a predictor in itself. Let call it $\left.\operatorname{Pred}_{R R B F}, 2\right)$ the complete NARX model composed of the RRBF and the AutoRegressive model. Let call it $\operatorname{Pred}_{\text {NARX }}$.

In Fig. 5, one can observe that the performances of the RRBF are significantly boosted by the uses of the non-linear NARX approach: predictions appear to be more accurate, precise, and reproducible: more closed to real values $(O A B)$, more grouped together $(O A V)$, and more independent from random initialization of the neural network parameters (Rep). This conclusion is reinforced by results shown in Fig. 6: the interest criterion of the Pred $_{N A R X}$ model is much higher than that one of the $\operatorname{Pred}_{R R B F}$ model (even if this comparison of the prediction models decreases as the horizon of prediction increases). More over, confidence on predictions increases whereas the effect of human intervention or of random initialization of parameters is much more reduced (the whole prediction system is more immune to initial parameterization). 


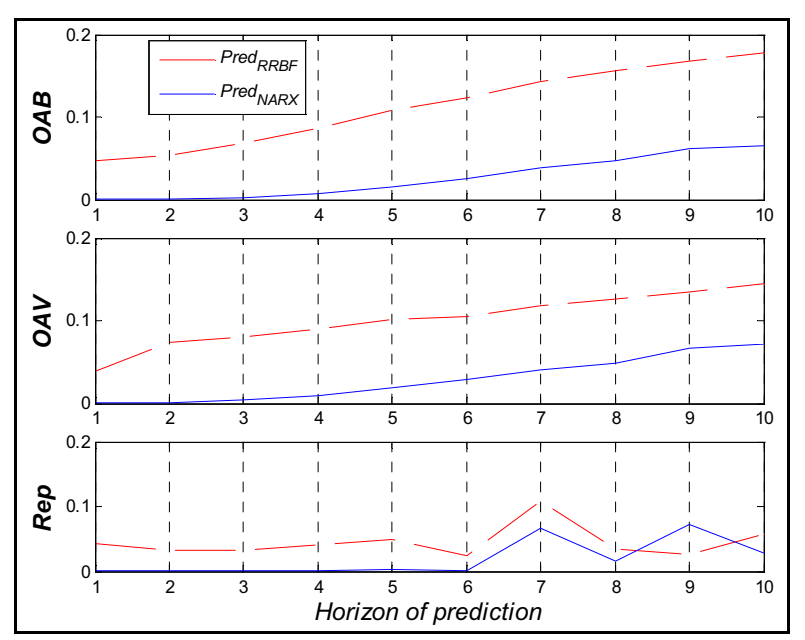

Fig. 5. Mackey Glass dataset - Prediction performances criteria: $O A B, O A V$ and Rep.

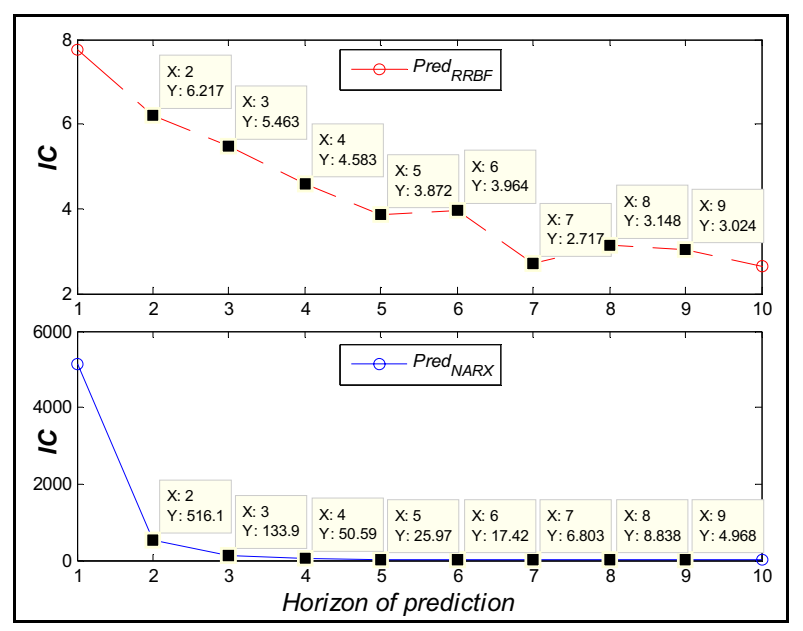

Fig. 6. Mackey Glass dataset - Interest criterion IC of models.

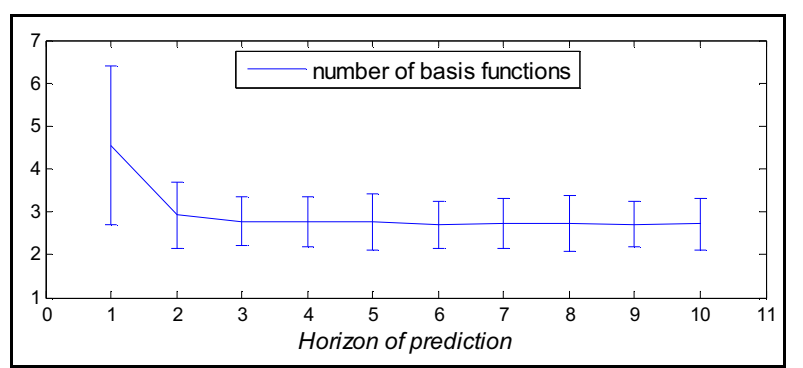

Fig. 7. Mackey Glass dataset - Complexity of the RRBF.

An other point of interest is that one of the complexity of the model and of the relative importance of the RRBF component with respect to the AutoRegressive process. Fig. 7 and 8 depict the number of basis functions of the RRBF and the evolution of the NARX parameters in (15) with regards with the horizon of prediction. That enable to judge from the complexity of the models. According to Fig. 7 the complexity of the RRBF is quite unchanged when the horizon of prediction increases. This result points out that there is no interest in trying to improve the accuracy of predictions by building more complex neural models. Linking it with the results depicted in Fig. 5 and 6, a better manner to build efficient prediction models is to complete the capability of the RRBF with approaches like this one proposed in this paper (by building a NARX model) in order to correct the error of prediction of the neural model. Finally, in Fig. 8, one can observe that the influence of the RRBF network seems to increase as the horizon of prediction does: the absolute values of parameters $a, b$, and $c$, that are related to the predictions of the RRBF (equation 15) grow with the horizon of prediction, whereas they turn around zero for short term predictions.

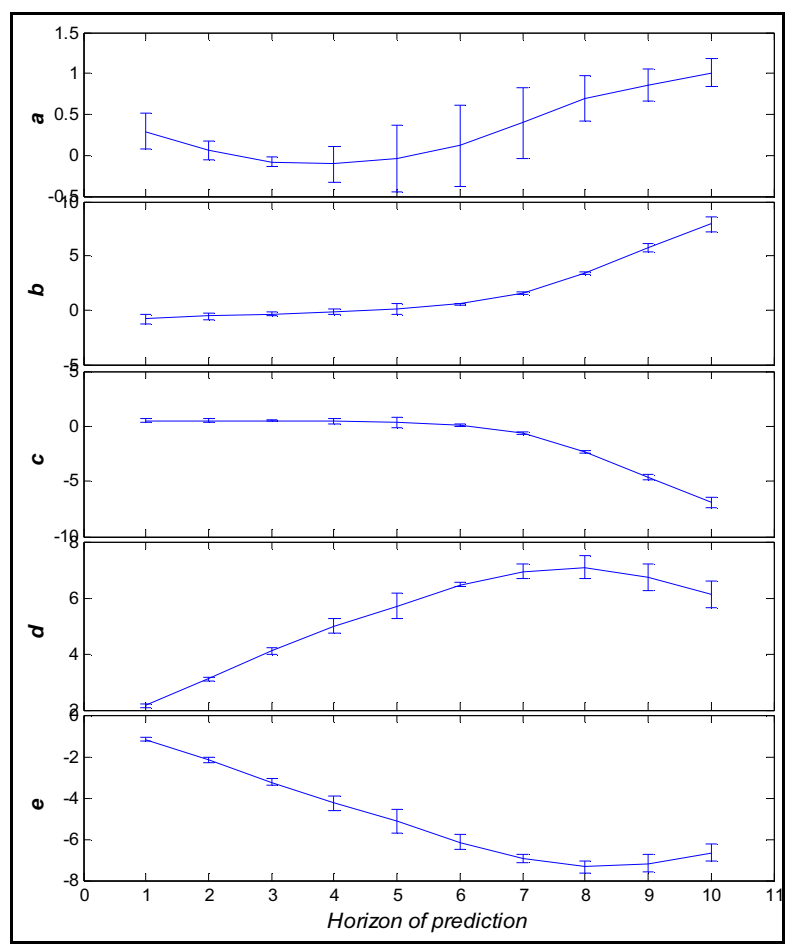

Fig. 8. Mackey Glass dataset - NARX parameters $a, b, c, d, e$.

\subsection{Second data set: prediction of an engine health}

The second illustration is made on the challenge dataset of diagnostic and prognostics of machine faults from the first International Conference on Prognostics and Health Management (Saxena et al., 2008). The dataset consisted of multiple multivariate time series (26 variables) with sensor noise (like in Fig. 9). Each time series was from a different engine of the same fleet and each engine started with different degrees of initial wear and manufacturing variation unknown to the user and considered normal. The engine was operating normally at the start and developed a fault that grew in magnitude until system failure.

For this paper, only feature number 3 has been considered. The same simulation procedure has been used as for the Mackey-Glass dataset: 10 data series were used for learning, 10 for validation and 10 for test. As for the regressors, both the $\operatorname{Pred}_{R R B F}$ and the $\operatorname{Pred}_{N A R X}$ models were built with the following inputs: $y(t)$ and $y(t-1)$. In order to limit the plan of test, and since the engine health is a huge dataset, the number $k$ centers (radial basis functions) has been set to 25 .

Results are shown in Fig. 10. Even if differences in between predictions obtained with the neural network model and the NARX model are not so significant as for the Mackey-Glass 
dataset, predictions appear to be improved with the proposed procedure: whatever the horizon of prediction is, $O A B, O A V$, Rep and $I C$ criteria are always slightly better with the NARX model. Note also that differences would have been greater if the number of $k$ centers would not have been fixed.

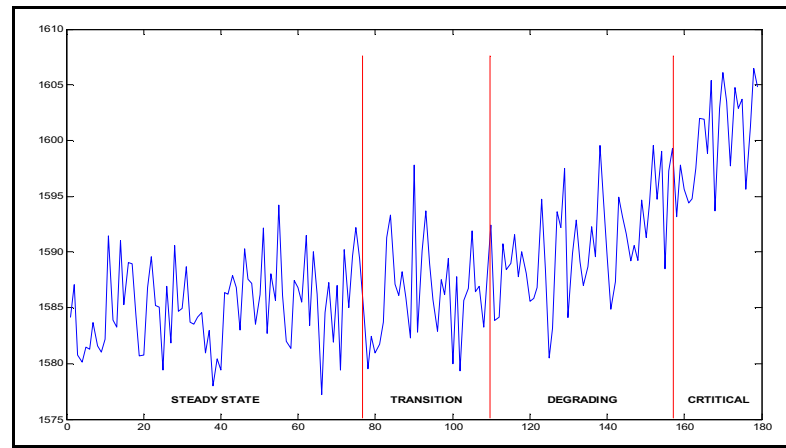

Fig. 9. Health engine dataset - Example of degradation.

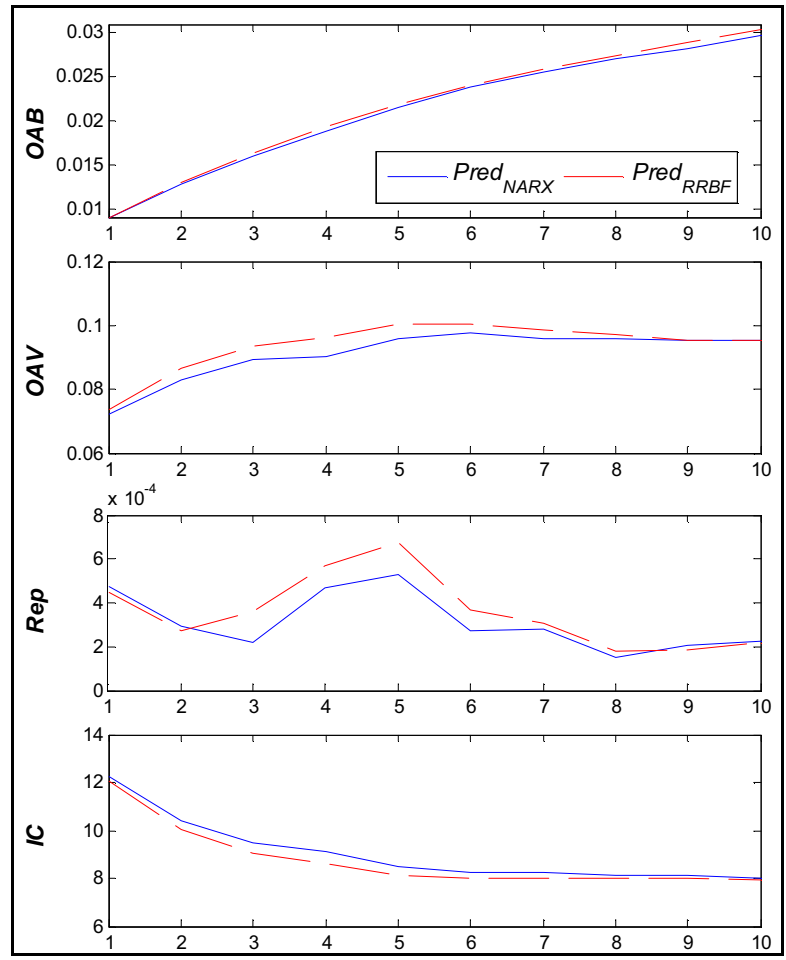

Fig. 10. Health engine dataset - Prediction performances and interest criteria: $O A B, O A V$, Rep and $I C$.

\section{CONCLUSIONS}

In maintenance field, prognostic is recognized as a key feature since the estimation of the remaining useful life of an equipment allows avoiding inopportune maintenance spending. However, it can be difficult to define and implement an adequate and efficient prognostic tool that includes the inherent uncertainty of the prognostic process. Indeed, an important task of prognostic is that of prediction. In this context, the purpose of the work of this paper is to point out an accurate prediction technique and to propose a way to improve its prediction performances. According to the global requirements that can be expected from a forecasting tool, the neural network RRBF has been presented as a candidate to support this activity. An improvement of this neural network has also been proposed by combining it with a AutoRegressive model. Moreover, since performances of an ANN are partially depending on an initial randomly parameterization of its structure, criteria have been proposed to quantify the precision, accuracy, and reproducibility of predictions. It enables to a priori estimate the usefulness of a neural network structure. Results show that the proposed prediction structure enables the forecasting to be a more robust task without increasing complexity of treatments. The whole is of good omen for prognostic purpose.

\section{REFERENCES}

Andalib, A. and Farid, A. (2009). Multi-step ahead forecasts for electricity prices using NARX: A new approach, a critical analysis of one-step ahead forecasts. Energy Conversion and Management, vol. 50:3, pp. 739-747.

Byington, C., Roemer, M., Kacprzynski, G. and Galie, T. (2002). Prognostic enhancements to diagnostic systems for improved condition-based maintenance. In Proc. IEEE Aerospace Conference, Big Sky, USA.

De Gooijer, J.G. and Hyndman, R.J. (2006). 25 years of time series forecasting. Int. J. Forecast., vol. 22, pp. 443-473.

Frasconi, P., Gori, M. and Soda, G. (1992). Local Feedback Multilayered Networks. Neural Computation, vol. 4, pp. 120-130.

Graves, D. and d Pedrycz, W. (2009). Fuzzy prediction architecture using recurrent neural networks. Neurocomputing, vol. 72:7-9, pp. 1668-1678.

ISO 13381-1 (2004). Condition monitoring and diagnostics of machines - prognostics - Part1: General guidelines. Int. Standard, ISO.

Jardine, A.K.S., Lin, D. and Banjevic, D. (2006). A review on machinery diagnostics and prognostics implementing condition-based maintenance. Mechanical Systems and Signal Processing, vol. 20, pp. 1483-1510.

Pisoni, E., Farina, M., Carnevale, C. and Piroddi, L. (2009). Forcasting peak air pollution levels using NARX models. Eng. Appl. of Artificial Intelligence, vol. 22, pp. 593-602.

Saxena, A., Goebel, K., Simon, D. and Eklund, N. Damage Propagation Modeling for Aircraft Engine Run-toFailure Simulation. In IEEE Prognostics and Health Management PHM'08 Conference, Denver CO, USA.

Vachtsevanos, G., Lewis, F.L., Roemer, M., Hess, A. and $\mathrm{Wu}$, B. (2006). Intelligent Fault Diagnosis and Prognosis for Engineering Systems. Wiley \& Sons, Hoboken, New Jersey.

Wei, H.L., Zhu, D.Q., Billings, S.A. and Balikhin, M.A. (2007). Forecasting the geomagnetic activity of the Dst index using multiscale radial basis function networks. Advances in Space Research, vol. 40:12, pp. 1863-1870.

Zemouri, R., Racoceanu, D. and Zerhouni, N., (2003). Recurrent Radial Basis Function network for TimeSeries Prediction. Eng. Appl. of Artificial Intelligence, vol. 16, pp.453-463.

Zemouri, R., Gouriveau, R., Zerhouni, R. (2009). Combining a recurrent neural network and a PID controller for prognostic purpose: A way to improve the accuracy of predictions. In PENTOM'09 Conf., Autrans, France. 